\title{
UN TÓTEM FRÁGIL: \\ APROXIMACIÓN A LA ESTRUCTURA \\ TEÓRICA DE EL SUICIDIO
}

\section{Ramón Ramos Torre}

Universidad Complutense

\begin{abstract}
RESUMEN
El Suicidio puede ser considerado como uno de los tótems de la sociología actual. Gran parte de su poder resulta de su densidad teórica. Pero su entramado teórico es complejo y está sometido a disputas interpretativas. Se propone reconstruir su lógica teórica como el resultado de un trayecto circular que inicialmente descompone analíticamente el objeto para después volver a él y hacerlo inteligible. Es en este trayecto de vuelta cuando aparecen serias aporías que enfrentan al principio de explicación causal con el principio de inteligibilidad de las prácticas analizadas. De ahí la fragilidad teórica de El Suicidio.
\end{abstract}

«Lo concerniente al totemismo tiene necesariamente repercusiones de alcance en todos los dominios de la sociología, pues se halla en la raíz de múltiples instituciones» (Durkheim, 1902a: 315-316). Así reflexionaba Durkheim en el volumen V del Année Sociologique, llamando la atención sobre la relevancia de la creciente literatura antropológica sobre el totemismo australiano. Hoy es lícito descontextualizar su aserto, olvidarse de los humildes Aruntas australianos a los que iba referido y proyectarlo reflexivamente sobre la empresa que Durkheim estaba entonces poniendo en marcha o incluso sobre su misma persona. Pues lo que el paso del tiempo nos ha mostrado es que la sociología misma se ha convertido en una institución que, como tal, hunde sus raíces en un tótem que "literalmente es condición de su existencia» (Durkheim, ibid.:

\section{Reis}


348), pues fija su fundación y modelo reverenciado. Tal tótem lo construyó el mismo Durkheim y apareció en las librería hace ahora un siglo bajo el nombre El Suicidio. Estudio de sociología. Humilde artefacto de papel, "tótem extraño» asegura alguno (Paul-Lévy, 1987: 243), tótem de lenta consagración pues, como ha mostrado Besnard (1987: 142 ss.), tuvo que sufrir un largo "purgatorio" hasta su reconocimiento a partir de los años cuarenta y, ciertamente, como veremos, tótem frágil: nada de esto impide que en la actualidad se reverencie como una de las obras fundacionales de la tribu contemporánea de los sociólogos.

Las razones del papel totémico de El Suicidio (SU) no son difíciles de fijar. Apuntaré algunas muy entrelazadas. La primera tiene que ver con la plena legitimación del proyecto sociológico. Obra valiente, SU se aventura en un arriesgado experimentum crucis para mostrar que el fenómeno aparentemente más individual e íntimo es abordable y explicable sociológicamente. Mostrada la viabilidad del proyecto en condiciones tan desfavorables, queda plenamente legitimado como ciencia clara y distinta ${ }^{1}$. La segunda razón se emparenta con la anterior. La significación totémica de SU proviene también de su éxito a la hora de acotar el objeto paradigmático de toda monografía sociológica. Muestra, en efecto, que el porvenir está en el estudio de hechos circunscritos, cuantificables, pero que, lejos de ser triviales, sean significativos socialmente, es decir, que operen como indicadores o reveladores de los niveles más opacos y decisivos de lo social ${ }^{2}$. Hay, además, una tercera razón que cualifica y da remate a las anteriores. Ya la puso de relieve Parsons al comentar: «hay muy pocas monografías en el campo de la ciencia social donde se combine tan felizmente los aspectos empíricos y teóricos» (Parsons, 1968: 385). Y es que la significación crucial de $S U$ responde a que es la primera muestra convincente de una sociología no sólo empíricamente rigurosa, sino sobre todo teóricamente informada y ambiciosa. Su genialidad no proviene tanto del tema escogido o del tratamiento estadístico que le proporciona, pues el tema era ya viejo en la reflexión social y la evidencia y correlaciones estadísticas ya habían sido exploradas $^{3}$. La innovación de Durkheim era de otro orden. Como subraya Obeschall (1990: 116), «el logro de Durkheim no fue metodológico. Utilizó la

1 Por otro lado, como destaca Giddens (1965: 10), no se trata tan sólo de legitimar genéricamente el proyecto sociológico, sino específicamente su versión durkheimiana. De ahí que en SU sea tan central la polémica con Tarde, representante de una visión alternativa de ciencia social menos polémica y circunscrita, más cercana a la psicología. Sobre la relación Durkheim-Tarde en el proceso de escritura de SU, véase Borlandi en este número de REIS.

2 Las conclusiones de SU son muy explícitas sobre este tema. Durkheim asegura que «una monografía sobre el suicidio tiene un alcance que va más allá del orden particular de hechos que aborda específicamente [...] Lo que prueba este número excepcional de muertes voluntarias es el estado de perturbación profunda que sufren las sociedades civilizadas, atestiguando su gravedad. Incluso se puede decir que mide su gravedad» (Durkheim, 1897: 450; énfasis RRT). Signo, testigo, medida de algo profundo, generalizado y grave: tal es el humilde hecho que se investiga.

3 Sobre el papel general de la estadística en la primera ciencia social anterior a Durkheim, véanse Daston (1990) y Oberschall (1990). Sobre las indagaciones en relación al suicidio anteriores a la publicación de SU, las correlaciones empíricas establecidas y sus líneas de interpretación se pueden consultar Giddens (1965) y Douglas (1966). 
misma técnica de comparación de las diferencias en tasas y correlaciones de uso común entonces. Tampoco descubrió nuevas y sorprendentes relaciones estadísticas entre el suicidio y otras variables. Lo que hizo fue proporcionar una explicación consistente de todas las relaciones estadísticas conocidas desde una perspectiva sociológica nueva».

El tótem que festejamos está, pues, teñido de teoría, de gran teoría. Sólidos intérpretes lo atestiguan. El ya citado Parsons (1968: 385) aseguró en su momento que, en la obra, "Durkheim consigue llegar a resultados que arrojan una luz sorprendentemente brillante sobre algunos de los problemas de más largo alcance de la teoría sociológica». En época más reciente, Alexander (1982: 228) ha llegado a decir que, en SU, Durkheim «está utilizando el suicidio como un caso paradigmático para la lógica teórica»; algo así como si el suicidio-tema fuera básicamente un buen pretexto para pensar. No resulta difícil suscribir comentarios tan entusiastas. Si SU brilla especialmente en la historia de la sociología es por su especial relevancia en el proceso de ruptura con aquella remota prehistoria en la que todavía la filosofía social y la estadística moral se encontraban disociadas - como muestra el rechazo del probabilismo estadístico por parte de Comte (Cohen, 1990: 38-39; Ewald, 1996: 108)—. La presencia de la teoría es lo que permite dar el paso para la superación de tal disyunción.

Es esto lo que me va a interesar en el desarrollo de este trabajo. $\mathrm{Si}$, por lo argumentado, podemos festejar el centenario de SU como un aquelarre totémico y si resulta que lo que da solidez a ese tótem es su especial textura teórica, entonces parece urgente proceder a indagarla aunque sólo sea con el propósito de saber más claramente a qué debemos la identidad de que, como sociólogos, empezamos a disfrutar hace un siglo. Que al final resulte que el tótem sea más frágil y problemático de lo que quisiéramos y esperábamos es lo de menos. Lo importante es saber cómo es y asumirlo piadosamente: un rey desnudo no deja de ser rey, por mucho que se mofen cuatro mocosos.

\section{INFLACIÓN INTERPRETATIVA}

Nadie se puede sorprender de que la literatura sobre la estructura teórica de SU sea voluminosa, desbordante. Obra leída y releída, enseñada en las aulas y comentada en innumerables trabajos escolares, ha acumulado sobre sí un ingente aparato crítico-interpretativo (Besnard, 1987: 48-81) que sería aquí ocioso revisar.

Pero no es ocioso comentar algo esperable y curioso a la vez. Me refiero al hecho de que, a la hora de fijar en qué consiste el discurso teórico de SU, esa literatura crítica carezca de un consenso básico. Este resultado es muy esperable porque, como es sabido, un texto, y tanto más si es crucial, queda en manos de sus múltiples lectores y, por lo tanto, de los interrogantes, prejuicios, informaciones y desinformaciones que les son propios. El juego de las múlti- 
ples lecturas es, pues, muy de esperar y hasta cabe celebrarlo como prueba de la riqueza semántica de un texto, incluso de su estatuto de clásico. Pero no deja de haber una cierta sorpresa en todo ello, pues lo que encontramos como lecturas auténticas en relación a un texto-tótem difieren tan radicalmente entre sí que lo convierten en una reliquia, ciertamente venerada, pero extraña, proteica, ambigua. ¿Cómo casar una lectura de SU en clave kantiana (La Capra, 1972: 165-166) con otra en clave schopenhaueriana (Mestrovic, 1987, 1988a y 1988b; Mestrovic y Brown, 1985)? ¿Cómo compatibilizar las propuestas que hablan, en múltiples versiones, de la existencia de dos variables ${ }^{4}$ del orden social con aquellas otras que proponen que Durkheim sólo utiliza una ${ }^{5}$ ? ¿Cómo decidirse por una versión subjetivo-voluntarista-idealista del orden social (Alexander, 1982: 225-233) frente a la más generalizada y evidente que resalta su carácter objetivista-determinista-materialista? Sobre todo, ¿cómo apostar por una línea interpretativa u otra cuando ambas están largamente documentadas y encuentran una amplia corroboración textual?

Resulta además que, lejos de desdibujarse, el problema se agudiza cuando de niveles interpretativos tan abstractos se pasa a niveles más epidérmicos. Y, en efecto, basta con observar la ingente literatura dedicada a la anomia — que tiene como único punto común filiarse en la obra de Durkheim, sobre todo en $\mathrm{SU}^{6}$ - para asistir al espectáculo de la hiperinflación semántica. Su significación se desborda de tal manera que, al final, puede significar cualquier cosa: todo, nada o «desarreglo [dérèglement]» (Mestrovic y Brown, 1985), «debilitamiento de la disciplina [de la conciencia colectiva]»(Parsons, 1968: 424), «quiebra de la estructura cultural [... por] disfunción aguda entre las normas y los objetivos culturales y capacidades socialmente estructuradas» (Merton, 1970: 170), «relajación de las regulaciones normativas»(Coser, 1971: 134), bajo "grado de integración normativa» (Giddens, 1966: 101), «alienación» (Srole, 1956: 711), etc. ${ }^{7}$.

${ }^{4}$ Evidentemente, esas dos variables — que siguiendo la propuesta terminológica dominante en Durkheim llamaré más adelante Integración y Regulación Sociales (véase Besnard, 1987)_ han sido denominadas y concebidas de forma muy variada: contenido de la conciencia colectiva vs fuerza de la conciencia colectiva (Parsons, 1968: 424), integración social vs integración normativa (Giddens, 1966: 101), consenso en los valores vs integración estructural (Coser, 1971: 132-136), etc.

5 Tal hace Halbwachs (1930: 502), tras reevaluar los datos de SU e intentar reformular y hacer más plausible la teoría de Durkheim. También lo proponen Johnson (1965: 886) o Pope (1976: 60) como lectura no textual de SU, pero acorde con la práctica analítica profunda de su autor.

6 Sobre la génesis del concepto de anomia desde el pensamiento clásico, pasando por Guyau, hasta Durkheim, véanse Orru (1983) y Mestrovic y Brown (1985). Sobre su deriva posterior tanto en la obra de Durkheim como en la de psicólogos y sociólogos de distinta orientación, véanse Horton (1964) y Mawson (1970) y, especialmente, el trabajo definitivo de Besnard (1978 y 1987).

${ }^{7}$ Llegando a lo humorístico, puede significar también «sin nombre» (wihout name), como se propone en el texto de J. Selkin (1982: 202), sin duda a resultas de un error tipográfico — ¿o no?, quién sabe... 
Esta primera aproximación muestra, pues, que lo que aquí se indaga es cuanto menos elusivo. Y lo es sin duda porque cualquier lector de SU podrá encontrar que, como tantas veces ocurre, lo que en la obra es decisivo no se muestra de forma clara y distinta. En efecto, la multiplicidad de líneas interpretativas es, por lo menos parcialmente, resultado de que Durkheim no presentara en ningún momento de forma clara y sistemática las propuestas teóricas fundamentales que hacían inteligible y explicable el suicidio como fenómeno social. No digo con esto que las ocultara, sino que, por las razones que fuera y en las que no entro, las presentó de una forma desordenada, a medio camino entre la dispersión y la reiteración inconsistente. La dispersión hace que el lector se vea abocado, tras múltiples relecturas, a poner juntas las piezas diseminadas, aquí y allá, del puzzle teórico. La reiteración inconsistente lleva a que la argumentación nunca aparezca acabada, cerrada en una formulación definitiva, sino que tienda a permanecer siempre abierta, introduciendo, al volver sobre un mismo tema teórico, nuevas matizaciones y deslizamientos que se sobreponen inflacionariamente. Resulta así que si en términos retóricos SU es un texto eficaz, compacto, que lleva al lector, en cómoda progresión, hacia una tesis final - a saber, el suicidio como hecho social y la sociología como ciencia competente para explicarlo-, en términos de su estructura teórica -que, aparentemente, tan decisiva es en ese juego retórico- es una obra oscura y requiere un lector muy atento que nunca encuentra la ayuda definitiva del inclemente Durkheim.

Lo anterior justifica la pertinencia de volver una vez más sobre la estructura teórica de SU, ampliando, para desesperación de durkheimiólogos, la caterva de las interpretaciones. No tengo la pretensión de dar con la interpretación definitiva, sino sólo de ampliar, en algunos aspectos, el universo de las interpretaciones posibles y plausibles. Al final quisiera mostrar que los problemas interpretativos de fondo no son artificiales. Responden al hecho de que Durkheim jugó con muchas barajas a la vez y generó un laberinto.

\section{EL CÍRCULO TEÓRICO}

Comenzaré la tarea presentando de forma muy sintética mi propuesta general y haciendo explícito el significado de los conceptos que utilizo.

Entiendo por estructura teórica el conjunto implícito o explícito de proposiciones cuyo cometido es hacer interpretable, inteligible y explicable un determinado objeto de estudio. La estructura teórica se apoya en, pero no se confunde con, las prescripciones metodológicas que permiten constituir un objeto y las técnicas de procesamiento de inscripciones objetuales que permiten cuantificarlo y compararlo en sus diferencias internas y externas. Por lo tanto, a la hora de fijar la estructura teórica de SU, dejaré en lo posible de lado los problemas de orden técnico-metodológico que le son característicos y que habrían de ser tratados aparte. 
La propuesta que hago es que, en una primera aproximación, la estructura teórica de SU es representable en forma de un círculo. En efecto, se desarrolla como un itinerario que parte de un punto de arranque para retornar finalmente a él. Ese punto de partida y llegada es el suicidio como hecho social, constituido como tal en razón de decisiones metodológicas. El trayecto que se sigue es, inicialmente, de orden analítico hasta alcanzar un estadio en el que se reorienta hacia cometidos directamente explicativos que lo hacen retornar al objeto de que había partido. Hay así como dos segmentos — analítico el uno, explicativo el otro- cuya unión da lugar al círculo completo del trayecto teórico. El primero es básicamente constructivo; el segundo aplica a un material el marco genérico construido, para lo que tiene que hacer elaboraciones parciales adicionales.

Entiendo por itinerario analítico el trayecto discursivo a lo largo del cual, en un proceso de progresiva abstracción, se van aislando los aspectos sociológicamente relevantes del objeto estudiado. Su cometido último es alcanzar proposiciones teóricas universales que den cuenta de las condiciones de posibilidad del orden social y, en razón de ello, de sus desviaciones patológicas. Alcanzado ese punto teórico, el itinerario se reorienta hacia la explicación propiamente dicha. Lo universal hallado ha de acomodarse a, y volcarse explicativamente sobre, una doble particularidad, la de lo histórico-social y la de lo puramente individual. Además, ha de abordar tanto la explicación de situaciones estructurales (escenarios) como de prácticas sociales (agentes). El acomodo significa que ha de hacerse cargo de las diferencias que muestran las sociedades humanas en su proceso de cambio histórico, pero también de las diferencias que muestran los individuos en sus prácticas concretas. Logrado el acomodo, ha de volcarse sobre ese material cumpliendo el cometido de explicarlo. Explicar significa que se ha de aislar las causas que actúan, así como hacer inteligible el modo en el que operan y, especialmente, la relación entre situación estructural (escenario) y práctica (agencia).

Fijada de forma muy sintética lo que considero la estructura teórica de SU, es preciso hacer una advertencia. Tal estructura es implícita y no se identifica con el orden de exposición de la obra. Quiero decir con ello que constituye una reconstrucción interpretativa que toma en consideración más la estructura mental del escritor de SU - lo que tiene que suponer para decir lo que dice; la secuencia de la lógica teórica subyacente- que el orden de plasmación de su escritura. Este orden de plasmación constituye el orden de exposición del texto, que debería ser analizado aparte y que se sitúa más bien en el plano de la retórica de SU. Pues todo texto es un acto comunicativo que pretende persuadir a un lector distanciado en el espacio-tiempo que puede no comprenderlo o rechazarlo. Como tal, está cargado retóricamente. Una de las técnicas persuasivas más potentes es la del itinerario progresivo, que arranca de un problema claramente circunscrito y va aproximándose a una meta final de plena inteligibilidad. El orden expositivo de SU sigue esa técnica. Entreverado en él se halla su estructura teórica, mucho más compleja y que tiene un formato circular. 


\section{SOBRE EL ITINERARIO ANALÍTICO}

El punto de partida no son ni los suicidios individuales ni lo que Durkheim, en función de las prescripciones metodológicas fijadas en Las reglas del método sociológico (RMS), define como suicidio en si $^{8}$, sino la tasa social de suicidios. Éste es el verdadero hecho social, que «no es una simple suma de unidades independientes [...] sino que constituye por sí mismo un hecho nuevo y sui generis, que tiene su unidad e individualidad y, en consecuencia, su propia naturaleza» (Durkheim, 1897: 8). Y es hecho porque es observable, exterior, y está dotado de permanencia o variabilidad pautada en el tiempo9. Del estudio de la tasa de suicidios arranca el itinerario analítico en su doble sentido de selección de aspectos y de abstracción teórica creciente. Los pasos son muy obvios para cualquier lector y se pueden enumerar así:

Primer paso: de la tasa de suicidios a sus variaciones sociales.

La labor tanto crítica - o de refutación de teorías rivales ${ }^{10}$ - como constructiva de Durkheim consiste inicialmente en fijar toda una serie de lo que suele denominar leyes sociales del suicidio, es decir, generalizaciones empíricas que muestran correlaciones firmes entre la variación de la tasa de suicidios y

8 Definición que, frente a las creencias de los actores y observadores ingenuos, «esté fundamentada objetivamente, es decir, corresponda a la naturaleza determinada de las cosas» (Durkheim, 1897: 2). Basada en la diferencia clásica doxalepisteme, la definición es contraintuitiva y se justifica operativamente (permite construir e incluir el tipo contraintuitivo de suicidio altruista). Con todo, la definición durkheimiana no consigue su cometido de «écarter systematiquement toutes les prénotions» (Durkheim, 1895: 30), ya que a lo largo de toda la obra a su autor no le queda más remedio que seguir y aceptar las definiciones sociales del suicidio (las que proporcionan los funcionarios públicos y se plasman en las estadísticas oficiales). El juego de la diferencia doxalepisteme se torna así en puro juego retórico con escasa proyección teórica.

9 Baudelot y Establet han destacado esta conversión tan característica de SU del registro estadístico en hecho social: "se llama hecho social a todo conjunto de acciones humanas cuya traza sobre un aparato de registro presenta una cierta regularidad, a saber: constancia cuando la sociedad (país, región, clase social, familia) no cambia; variación reglada y definida cuando distintas magnitudes sociales varían simultáneamente» (Baudelot y Establet, 1984: 23). En razón de esto, en las páginas finales de SU, Durkheim asegura que la objetividad de los hechos sociales «encuentra en la estadística moral y sobre todo en la del suicidio una prueba adicional y particularmente demostrativa» (Durkheim, 1897: 349). De este modo, lo que es un presupuesto metodológico se convierte en una demostración a partir de los hechos. Por lo demás, al proponer esto, Durkheim no hacía sino seguir la estela de los estadísticos morales desde los tiempos de Quetelet —véanse Daston (1990) y Oberschall (1990).

${ }^{10}$ Esta labor, que se realiza a lo largo del Libro I de SU, es de una enorme relevancia para fundamentar el presupuesto de que la tasa de suicidios es un hecho social que, en consecuencia, sólo se puede explicar sociológicamente. El formato de la argumentación es el que proporciona el argumentum per eliminationem, como se evidencia en las páginas iniciales del Libro II: descartadas las causas físicas, biológicas y psicológicas, asegura Durkheim, «resulta por eliminación que debe necesariamente depender de causas sociales y constituir por sí misma un fenómeno colectivo" (1897: 139). Sobre la importancia de este y otros "vicios» lógicos en la retórica de Durkheim, véanse Lukes (1984: 31-35) y Overington en este volumen. 
un número de variables sociales ${ }^{11}$. A éstas las suele denominar sociedades y su lista no es muy amplia: sociedades religiosa, familiar (conyugal y paternofilial), política, económica, militar. En el seno de cada una de ellas distingue tanto situaciones procesuales (crónicas o coyunturales ${ }^{12}$ ) como, sobre todo, papeles, roles o tipificaciones sociales diferenciados (sacerdote/lego, oficial/suboficial, casado/soltero/divorciado/viudo, profesión agrícola/industrial, etc). Esta última distinción permite unir las variaciones sociales de la tasa de suicidios con las conductas individuales en función de sus tipificaciones sociales.

En este primer paso la labor de Durkheim es doblemente analítica. Por un lado, separa o segrega aquellos aspectos de las variaciones de la tasa social de los suicidios que son relevantes sociológicamente. Por otro lado, discrimina aquellos aspectos de los actores-suicidas que resultan decisivos y eventualmente explicativos desde un punto de vista sociológico. En razón de ambas labores de disección de lo concreto-inmediato, se procede en términos de creciente abstracción, polarizando la atención en los aspectos genéricos o supraindividuales (ser católico, oficial en el ejército, soltero/casado, joven/viejo, divorciado/a, etcétera).

Segundo paso: de las variaciones sociales a los tipos sociológicos de suicidio.

El siguiente paso reúne en sus especies fundamentales las distintas variaciones legaliformes anteriormente fijadas. La propuesta de Durkheim es que no son las características individuales de las distintas sociedades las que eventualmente pueden dar razón de las variaciones de la tasa de suicidios ya exploradas, sino ciertos rasgos comunes y, por lo tanto, más genéricos. Surge así la tipología cuatripartita ${ }^{13}$ que diferencia egoísmo, altruismo, anomia y fatalismo. Lo que se propone es que, desde un punto de vista sociológico, cualquier práctica suicida es un caso de uno de estos tipos o una combinatoria compleja de ellos $^{14}$. Los tipos no reciben definiciones canónicas circunscritas y definitivas,

11 Este concepto de ley impregna el lenguaje cientifista de los estadísticos morales de la época, a los que Durkheim sigue en este punto. Sobre la semántica de la ley social en la obra de Quetelet, véanse Lécuyer (1990: 321) y Ewald (1996: 108 ss.).

12 Situaciones coyunturales como las crisis políticas, bélicas o económicas (Durkheim, 1897: 215-222, 264-271); situaciones crónicas como la que caracteriza a la economía moderna (ibid.: 282-288).

${ }^{13}$ La tipología no es siempre reconocida como cuatripartita, habiendo una tendencia en la literatura crítica a dejar de lado el fatalismo. La razón es obvia ya que este tipo apenas queda esbozado en una nota a pie de página que cierra el extenso capítulo sobre el suicidio anómico. Con todo, un estudio sistemático sobre la estructura teórica de SU ha de tomar en consideración los cuatro tipos con independencia de su relevancia empírica. Sobre la relevancia del fatalismo y las razones de su infrautilización por Durkheim, véase Besnard (1973).

${ }^{14}$ Lo que denomina tipos mixtos son explorados en el capítulo que cierra el Libro II, cuando se pasa de la tipología etiológica a la tipología morfológica y, con ella, a las formas individuales que revisten los tipos sociales. Véase el cuadro sistemático final («clasificación etiológica y morfológica de los tipos sociales del suicidio") (Durkheim, 1897: 332), donde se toman en consideración distintas combinaciones de anomia, altruismo y egoísmo. 
sino que su delimitación semántica queda difusa en razón de las estrategias textuales antes mentadas de la dispersión y la reiteración argumentales ${ }^{15}$.

Este segundo paso refuerza la estrategia analítica. Lo propio y distintivo de las distintas «sociedades» queda cancelado a favor de aspectos comunes que responden a, y son subsumibles bajo, los conceptos abstractos de egoísmo, altruismo, anomia o fatalismo. En razón de ello, tanto la tasa de suicidios como las conductas individuales que refleja quedan insertadas en un nivel teórico de pretensiones universalistas cuyos fundamentos y significación son aclarados en el siguiente paso analítico.

Tercer paso: de los cuatro tipos a las dos variables sociológicas estratégicas.

La clave que justifica la tipología cuatripartita la proporciona el aislamiento de las dos variables sociológicamente relevantes. Aunque su denominación no es todo lo firme que sería de agradecer, aparecen fijadas como Integración y Regulación sociales. La especificación de su semántica sufre de nuevo de la estrategia de dispersión y reiteración antes mentada, oscilando entre enunciados contextuales y presentaciones metafóricas ${ }^{16}$. En cualquier caso, las dos variables actúan como matriz de los cuatro tipos en razón de un principio de simetría que se hace plausible a la luz de la teoría de lo Normal/Patológico que

15 Para la acotación semántica de los tipos, véanse notas 16 y 17.

${ }^{16}$ La metáfora dominante que intenta hacer representable lo que resulta difusamente conceptuable es de orden físico, basada en la diferencia entre atracción y contención-presión. Y así: «La sociedad no es sólo un objeto que atrae hacia sí con desigual intensidad los sentimientos y la actividad de los individuos. Es también un poder que los regula [règle]» (SU: 264). La integración opera en el orden de la atracción; la regulación, en el orden de la contención-presión. Desde un punto de vista más conceptual, ambos conceptos se refieren tanto a las características estructurales de un grupo o del conjunto de grupos que conforman una sociedad como a las relaciones de los individuos con ambos. En razón de esto pueden estar integrados/regulados un grupo o los individuos en/por el grupo. La semántica de ambos conceptos se logra reconstruir por acumulación de especificaciones locales (contextos concretos de análisis) que atienden a sus indicadores sociales. En razón de ello cabe proponer las siguientes delimitaciones:

Integración = estado de cohesión de la estructura social y de las relaciones entre ésta y los individuos que varía en función de

- el número de creencias y prácticas comunes y obligatorias (análisis local: sociedad religiosa);

- fines colectivamente asumidos y perseguidos (análisis local: sociedad política);

— densidad comunicativa interior a un grupo (análisis local: sociedad familiar).

Regulación = estado de control de la estructura social y de las relaciones entre ésta y los individuos que varía en función de

- el ámbito de la definición normativa de fines y medios (análisis local: situación crónica de la sociedad económica);

- la estabilidad y legitimidad de los códigos normativos (análisis local: crisis coyunturales de la sociedad económica);

— la solidez o confiabilidad de los códigos normativos (análisis local: sociedad conyugal). 
más adelante se comentará. Distinguiendo entre situaciones polares Hiper(exceso) e Hipo- (defecto) de la integración y regulación sociales, Durkheim proporciona el fundamento analítico de los cuatro tipos de suicidio ${ }^{17}$. La matriz teórica simple es la recogida en el siguiente cuadro 1 .

\section{CUADRO 1}

\section{Modelo analitico simple}

\begin{tabular}{c|c|c|}
\hline \multirow{2}{*}{ INTEGRACIÓN } & EXCESO & DEFECTO \\
\cline { 2 - 3 } & ALTRUISMO & EGOÍSMO \\
\cline { 2 - 3 } REGULACIÓN & FATALISMO & ANOMIA \\
\hline
\end{tabular}

La labor analítica (separación y abstracción) realizada no es, con todo, definitiva ya que su resultado sólo es comprensible si se toma en consideración lo que es propio del cuarto y último paso del itinerario analítico, en el que se alcanza el máximo de abstracción y, consecuentemente, de universalidad de las proposiciones teóricas.

Cuarto paso: de las variables a las condiciones de posibilidad del orden social.

Partiendo de un fenómeno biológica y socialmente patológico, Durkheim ha procedido a reconducirlo al marco analítico de la Regulación e Integración

17 La semántica de los tipos se fija, pues, a partir de la distinción exceso/defecto aplicada a la semántica de la regulación e integración sociales. Y así resulta:

Egoísmo: integración defectuosa que limita desmesuradamente la cantidad e intensidad de creencias y prácticas comunes y obligatorias, y/o de fines colectivos, y/o de densidad comunicativa (aislamiento).

Altruismo: integración excesiva que expande desmesuradamente la cantidad e intensidad de creencias y prácticas comunes y obligatorias, y/o de fines colectivos, y/o de densidad comunicativa.

Anomia: regulación defectuosa que limita desmesuradamente el ámbito de la definición normativa de fines y medios, y/o la estabilidad y legitimidad de los códigos normativos, y/o su solidez o confiabilidad.

Fatalismo: regulación excesiva que expande desmesuradamente el ámbito de la definición normativa de fines y medios, y/o la estabilidad y legitimidad de los códigos normativos, y/o su solidez o confiabilidad.

Los distintos tipos definen tan sólo desviaciones normales del orden social. Generan situaciones patológicas según el defecto o el exceso se va agudizando y expandiendo a ámbitos más amplios de la vida social. 
sociales. Situado en tal marco, puede entonces proceder a enunciar las condiciones de posibilidad de todo orden social que son, lógicamente, previas a cualquier tipificación de patologías o anormalidades. El enunciado general es que el orden social se hace posible en función del grado de Integración y Regulación sociales. Cuando se alcanza un grado de equilibrio en ambas variables se dará una situación de orden. Cuando, por el contrario, haya excesos o defectos de integración y/o regulación surgirá una situación de desorden que se mostrará estadísticamente en incrementos de la patología social — sea en forma de suicidios o de otras prácticas sociales-. El cuadro 2 resume la propuesta.

\section{CUADRO 2}

Cuadro analitico sobre las condiciones de posibilidad del orden social

\begin{tabular}{l|c|c|c|}
\hline \multicolumn{1}{c}{ EXCESO } & \multicolumn{2}{c}{ EQUILIBRIO } & \multicolumn{1}{c|}{ DEFECTO } \\
\cline { 2 - 5 } INTEGRACIÓN & ALTRUISMO & $\begin{array}{c}\text { INTEGRACIÓN } \\
\text { ADECUADA }\end{array}$ & EGOÍSMO \\
\cline { 2 - 5 } REGULACIÓN & FATALISMO & $\begin{array}{c}\text { REGULACIÓN } \\
\text { ADECUADA }\end{array}$ & ANOMIA \\
\hline
\end{tabular}

Por otro lado, como en cualquier situación social concreta operan ambas variables, habrá que considerar la combinación de los distintos estados de Integración y Regulación sociales. Aunque no explorada sistemáticamente en las páginas de $\mathrm{SU}$, tal posibilidad genera la combinatoria compleja recogida en el cuadro 3, que sigue con algunas variaciones el propuesto por Johnson (1965).

En esta tupida red se consideran las distintas situaciones de orden y desorden sociales. Siendo la situación numerada 5 la de perfecto equilibrio y, por lo tanto, de pleno orden, y las situaciones 1, 3, 7 y 9 de máximo desequilibrio y, consecuentemente, de aguda patología, las situaciones 2, 4, 6 y 8 retratan desequilibrios sólo parciales y, por ello, patologías benignas.

El resultado alcanzado supone el punto definitivo de llegada del itinerario analítico desarrollado a partir de la fijación de la tasa social de suicidios como hecho social a estudiar. El marco teórico construido tiene pretensiones de universalidad, es decir, no se propone para dar cuenta de específicos fenómenos circunscritos espacial o temporalmente, sino de cualquier fenómeno social que se analice desde la perspectiva de la distinción normal/patológico. Como tal, hace abstracción de diferencias socioevolutivas entre sociedades y de diferencias sociales entre prácticas individuales. En razón de ello hay que considerarlo 


\section{CUADRO 3}

Cuadro analítico complejo

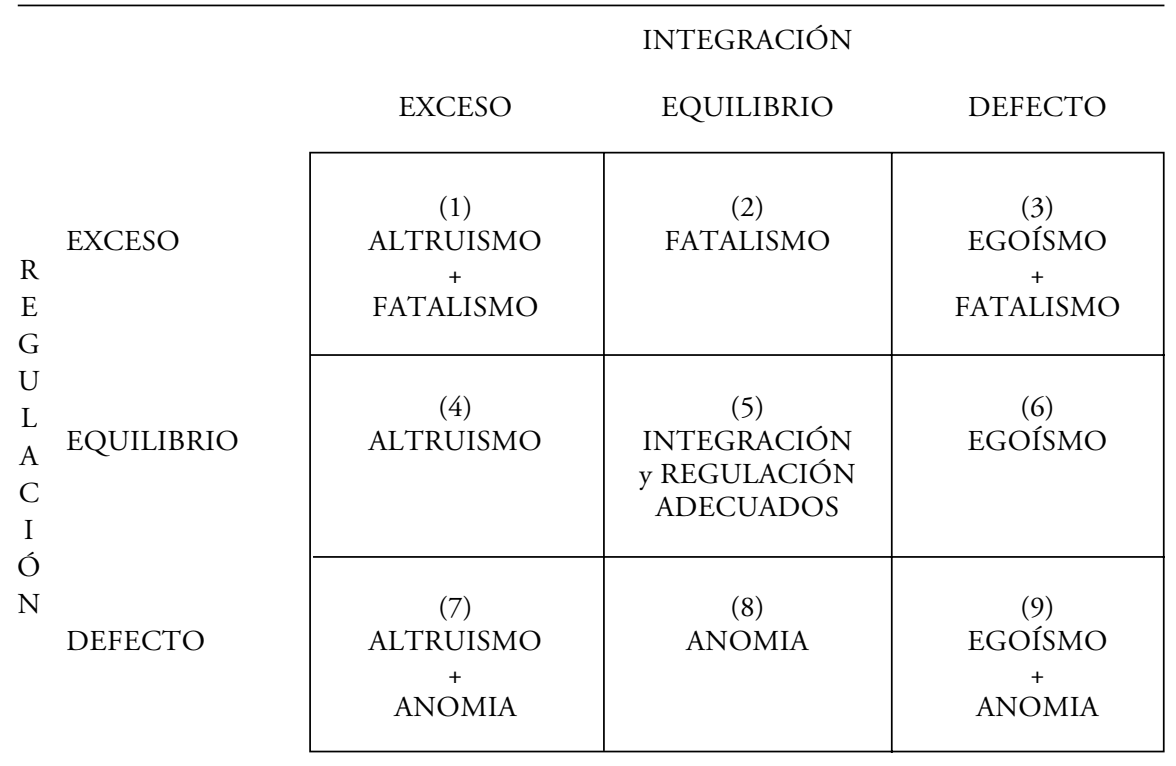

más como punto de arranque de la explicación que Durkheim quiere alcanzar sobre las variaciones históricas e individuales de la práctica suicida que como terreno explicativo ya alcanzado. De ahí que sea preciso invertir la marcha del itinerario para realcanzar, tras el logro de la plena explicación, el punto de partida y objeto de estudio: la tasa social de suicidios.

\section{SOBRE EL ITINERARIO EXPLICATIVO}

Ese viaje de vuelta al origen lo emprende Durkheim en pos de la explicación. Entiendo que una explicación propiamente dicha sólo se da cuando se cumplen dos condiciones: la condición de causalidad que establece, a partir de alguna regularidad secuencial, un nexo entre dos o más fenómenos, y la condición de inteligibilidad que establece qué mecanismo hace inteligible ese nexo causal $^{18}$. Podemos suponer que si Durkheim pretende explicar sociológicamen-

18 Esta distinción aparece en Elster (1991: cap. 1) en el marco de su crítica general al funcionalismo. Con todo, como ha mostrado Van Parijs (1990), esta distinción no es contradictoria con el funcionalismo en general, sino sólo con alguna de sus variantes explicativamente más perezosas. 
te las variaciones de la tasa de suicidios ha de emprender - explícita o implícitamente - la doble tarea de fijar las causas y hacer inteligibles cómo operan éstas sobre las conductas de los agentes que realmente se suicidan. Para que tal explicación sea teóricamente plausible, ha de hacerse en el marco del entramado analítico que se acaba de reconstruir, agregando las especificaciones que se consideren pertinentes pero sin contradecirlo o negarlo.

El explicandum es obvio: las variaciones en la tasa de suicidio. Pero ese objeto tiene al menos dos significaciones que, a lo largo de las páginas de SU, Durkheim se ha esmerado en resaltar. Por un lado, hace referencia a agregados de conductas individuales que, en razón de su permanencia a lo largo del tiempo, se muestran como hechos sui generis. Esto lleva a que, de alguna manera, haya que explicar de qué modo la pura agregación estadística de conductas individuales se convierte en un hecho social, más allá de prescripciones metodológicas. Pero, por otro lado, Durkheim presenta las variaciones de la tasa de suicidio como un exponente, signo o manifestación de algo que está más allá: el malestar social característico de una determinada sociedad ${ }^{19}$. En razón de esto, la indagación se abre más ampliamente hacia la explicación del malestar social y de sus variaciones socioevolutivas.

Evidentemente, las dos caras del explicandum están relacionadas. Explicar por qué se suicida la gente de forma tan regular es también explicar las fuentes del malestar social, por lo menos si consideramos lo uno como indicador de lo otro. Pero conviene distinguir ambas tareas ya que pudiera resultar que no dieran lugar a idénticos resultados. Empezaré reconstruyendo los argumentos de Durkheim cara a la explicación del malestar social.

Consciente de los cambios sufridos por las sociedades humanas a lo largo del tiempo, Durkheim traduce su marco analítico general en términos históricos. Como las condiciones de posibilidad del orden social dependen siempre de la conformación de los mecanismos de integración y regulación sociales, las distintas variantes históricas del malestar social habrán de ser reconducidas a esa matriz. Como, por otro lado, el proceso de cambio histórico — tal como había sido teorizado en De la división del trabajo social (DTS $)^{20}$ — no es sino el paso evolutivo hacia una mayor diferenciación social (paso de la solidaridad mecánica a la solidaridad orgánica), entonces es lógico distinguir dos situaciones polares de malestar social correspondientes a los dos tipos evolutivos que se distinguen. El resultado se plasma en el diagnóstico evolutivo sobre el malestar social que aparece en el cuadro 4.

19 Véase nota 2.

${ }^{20}$ Sabido es que las relaciones entre las tesis de DTS y SU no son de pura continuidad. Es llamativo y significativo que Durkheim no emplee el concepto de solidaridad social en SU. Con todo, el discurso evolucionista se mantiene en SU — como, por lo demás, en el conjunto de la obra de Durkheim-, donde se insiste en la lógica de la diferenciación y en la distinción entre las sociedades tradicionales basadas en la homogeneidad y las sociedades civilizadas basadas en la diferenciación y sus consecuencias características: el progreso y el individualismo. Esto hace legítimo la utilización del esquema bipartito de DTS en este contexto. 


\section{CUADRO 4}

Modelo histórico

\begin{tabular}{|c|c|c|}
\hline & $\begin{array}{l}\text { SOLIDARIDAD } \\
\text { MECÁNICA }\end{array}$ & $\begin{array}{l}\text { SOLIDARIDAD } \\
\text { ORGÁNICA }\end{array}$ \\
\hline INTEGRACIÓN & ALTRUISMO & EGOÍSMO \\
\hline REGULACIÓN & FATALISMO & ANOMIA \\
\hline
\end{tabular}

El resultado parece mimetizar el que se alcanzaba en el cuadro 1, pero en realidad introduce diferencias tajantes. Pues lo que se sostiene es que, en el marco de las posibilidades patológicas fijadas en el modelo analítico general, en los distintos tipos de sociedad se activan patologías distintas (de ahí la doble línea vertical de separación que se introduce en el cuadro 4). Lo propio de nuestras sociedades es el malestar que proviene de conformaciones Hipo(defecto) tanto de la regulación como de la integración sociales ${ }^{21}$. En razón de ello son sociedades de patología anómico-egoísta y se puede afirmar, sin resto de ambigüedad, que ambas fuentes del malestar no sean sino las dos caras de una misma situación o "dos aspectos diferentes de un mismo estado social» (Durkheim, 1897: 325) ${ }^{22}$.

Establecida la proyección histórico-evolutiva del marco analítico general, cabe entonces proceder a la explicación de las transformaciones del malestar social. Coherente con sus tesis generales sobre la patología social23, Durkheim

${ }^{21}$ Y, así, Durkheim (1897: 259-260) sostiene que los casos presentes de suicidio altruista son legados evolutivos de situaciones sociales anteriores e irán desapareciendo según las transformaciones evolutivas afecten a todas las esferas sociales. Lo mismo sostiene en la corta nota en la que presenta el suicidio fatalista (ibid.: 311). Su argumentación en este caso se ampara en el más estricto patriarcalismo ya que supone implícitamente considerar a los jóvenes y las mujeres como fósiles evolutivos apenas civilizados. Sobre estas implicaciones del fatalismo, véanse Besnard (1973), Tiryakian (1981), Ramos (1996).

22 Tesis que no se debe interpretar, al modo de Johnson (1965) y Pope (1976: caps. 4 y 5), como la admisión explícita de la indistinción de ambos tipos de patología social.

${ }_{23}$ Tesis que, tomando en consideración lo que se argumenta en RMS (Durkheim, 1895: cap. 3) y se reitera de forma comprimida en SU (Durkheim, 1897: 413-424), se pueden resumir así (Ramos, 1982: 54-55): a) ningún fenómeno social es en sí mismo patológico (no lo son ni el crimen ni el suicidio); b) lo patológico no es sino una forma extrema (por defecto o por exceso) de algo en sí normal (las manifestaciones de la regulación e integración sociales); c) lo patológico es simplemente una tasa excesiva de un fenómeno en sí normal —en el sentido fijado en $a$ ), pero con las características de $b$ )—. Si en a) y c) la distinción implícita normal/patológico es puramente estadística (frecuente $v$ infrecuente), en $b$ ) es además valorativa (bueno $v$ s malo). El paso de lo uno a lo otro — es decir, de la normalidad estadística a la normalidad valorativa - llena de 
propone que el malestar moderno no proviene de la anomia y el egoísmo en sí mismos, sino de su tasa excesiva. Una cierta anomia y un cierto egoísmo son inseparables de sistemas sociales que, en términos de regulación, optan por el progreso - y por lo tanto problematizan los códigos normativos establecidos (anomia) - y, en términos de integración, se conforman alrededor del culto colectivo a la persona humana o individualismo - y por lo tanto han de dejar espacios cada vez más amplios al libre examen (egoísmo)—. Anomia y egoísmo no son sino consecuencias normales del progreso y el individualismo modernos $^{24}$, pues cada tipo sociológico no es sino la "forma exagerada o desviada de una virtud» (Durkheim, 1897: 263). El malestar moderno proviene de su tasa excesiva. Tales excesos de anomia y egoísmo son ya fenómenos patológicos y hay que explicarlos como efecto de un proceso excesivamente acelerado de cambio social, que ha destruido los viejos cuadros sociales e impedido la correcta operación de los mecanismos de la integración y regulación sociales. Tal es el argumento dominante en la parte final de SU.

La explicación que proporciona Durkheim no genera especiales dificultades con tal de que se acepten sus presupuestos evolucionistas. Éstos dictan que el orden social se genera en el tiempo en un proceso socioevolutivo que se desliza entre dos situaciones polares: punto de partida y punto de llegada. Ambas suponen una conformación correcta del orden social. En razón de ello es esperable que los procesos transicionales sean especialmente arriesgados y que, en función de la tasa de cambio, se haga posible la aparición de sociedades que todavía no estén plenamente constituidas y carezcan de los mecanismos de regulación e integración propios de un tipo estable. En consecuencia, toda patología ha de concebirse como transicional y bastará con la construcción social de los nichos regulador-integradores correspondientes para que haya instituciones sociales adecuadas. Las propuestas finales de SU sobre las corporaciones tienen este sentido constructivo ${ }^{25}$.

No entraré en la evaluación de estas propuestas que, en sus distintas versiones, tanto éxito han obtenido en las ciencias sociale ${ }^{26}$. Valen lo que sus presu-

complejidad semántica la diferencia normal/patológico. Si, por una lado, la estadística neutraliza el mal (Ewald, 1996), el deslizamiento hacia los valores lo reintroduce como sociológicamente relevante. Y así, la sociología durkheimiana se ve abocada a remoralizar lo que ha desmoralizado.

${ }^{24}$ No hay identidad, sino semejanza. Las tesis de Durkheim son inequívocas. Si, por un lado, asegura que «el individualismo no es necesariamente el egoísmo, pero se le aproxima [y que] no se puede expandir el uno sin el otro", por el otro, se destaca que "toda moral de progreso y perfeccionamiento es inseparable de un cierto grado de anomia» (Durkheim, 1897: 416-417).

${ }^{25}$ El tema se aborda en las páginas finales (Durkheim, 1897: 434 ss.). La crisis de las instituciones tradicionales (familia, religión, etc.) no puede ser resuelta en términos de restauración de un pasado definitivamente superado, sino en términos de instauración de instituciones nuevas, como son las corporaciones que conseguirán «multiplicar los centros de vida común» (ibid.: 449) y superar el resultado coyuntural del desarrollo histórico que «ha hecho sucesivamente tabla rasa de todos los antiguos marcos sociales» (ibid.: 446). Sobre esta propuesta volverá Durkheim, en términos más sistemáticos, en el prólogo a la 2. ${ }^{\mathrm{a}}$ edición de DTS.

${ }^{26}$ Hasta el punto de constituir el equipamiento intelectual de la sociología del cambio. Para una exposición y crítica, véase Tilly (1991: cap. 1). 
puestos evolucionistas y son coherentes con, y se acomodan sin problemas a, el marco analítico anteriormente reconstruido. Más relevantes me parecen las propuestas de SU cuando se aborda esa segunda cara del explicandum a que antes hacía referencia. ¿Cómo explicar las prácticas de los individuos que se agregan estadísticamente y generan un hecho sui generis?

La respuesta a este interrogante es mucho más compleja que la que se acuerda al anterior. Las razones son dos. La primera es que en este caso hay que operar en los dos lados de una diferencia que, aunque implícita en la indagación sobre el malestar social genérico, podía entonces ser eludida. Me refiero a la diferencia situación/agente. Digo que estaba implícita porque, obviamente, el malestar moderno habla de situaciones y de prácticas de agentes. Pero está eludida porque en el nivel tan general de diagnóstico en el que operaba Dur-kheim podía ceñirse exclusivamente a un discurso cerradamente estructural que sólo mentara las situaciones sociales de anomia y egoísmo, dando por supuestas las prácticas agenciales correspondientes. Por el contrario, al abordar las prácticas individuales que se agregan estadísticamente y generan un hecho sui generis, Durkheim no puede eludir la distinción situación/agente y ha de dar cuenta de ambas caras.

La otra razón de la complejidad teórica creciente dimana de lo que anteriormente se presentó como doble condición de la explicación. Ésta ha de proporcionar causas que puedan operar como tales, es decir, que aclaren también un principio de inteligibilidad que muestre de qué manera operan sobre sus efectos. Por lo tanto, si se parte de las situaciones como causas habrá de establecerse cómo operan sobre los agentes para hacer inteligible que éstos actúen de la manera que lo hacen.

Abordando y desbrozando este complejo de distinciones, Durkheim propone explicar la práctica suicida combinando dos argumentos: uno se centra en las corrientes sociales que actúan como "causas suicidógenas» (Durkheim, 1897: 337); el otro en la idea de la naturaleza dual del hombre. El primer argumento centra su atención en la lógica de la situación y cumple de forma estricta con la condición de causalidad propia de todo esquema explicativo. El segundo argumento se adentra en las características cruciales del agente que realiza las prácticas y responde a los requerimientos de la condición de inteligibilidad. El problema que Durkheim no sabrá resolver es el de la relación entre ambos argumentos, generando un hiato explicativo insalvable que acabará creando tupidos laberintos, como se verá en su momento.

Las corrientes sociales son sedimentaciones de muy baja densidad de la vida social ${ }^{27}$. Producto de la vida en grupo en igual medida que los objetos materiales y las instituciones, actúan como verdaderas fuerzas. Durkheim ase-

27 Ya en el capítulo primero de RMS se había destacado la heterogeneidad de los hechos sociales, que forman un espectro con distinto grado de materialización o cristalización. En SU se retoma el tema, distinguiendo las formas materiales, las formas institucionales y las corrientes de opinión — vida social «difusa [...] que queda en libertad [...] y carece del suficiente grado de consistencia para fijarse» (Durkheim, 1897: 355-356). 
gura que son «tan reales como las fuerzas cósmicas, aunque tengan otra naturaleza»; subraya que "actúan también sobre el individuo desde fuera, aunque por otras vías", y advierte que su intensidad se puede medir "como se hace con la intensidad de las corrientes eléctricas» (ibid.: 348-349). Pueblan, pues, el entorno externo en el que se desarrollan las prácticas humanas, al lado de, y con la misma «realidad» que, el resto de las fuerzas cósmicas. Y, poblándolo, actúan sobre esas prácticas conformándolas, arrastrándolas, "como conjuntos energéticos que determinan nuestra acción desde fuera, del mismo modo que lo hacen las energías físico-químicas cuya acción sufrimos» (ibid.: 349).

Esta reafirmación de la teoría del hecho social como exterioridad constrictiva, observable y medible - lo que ya Knapp, crítico de Quetelet y su homme moyen, había denominado "concepción astronómica de la sociedad» (citado en Porter, 1990: 366) — fundamenta la explicación causal de las prácticas suicidas. La conocida tesis que Durkheim reitera a lo largo de la última parte de SU es que la patología estructural (defectos en la conformación de los mecanismos de regulación e integración sociales) se traduce en corrientes sociales de malestar que penetran en los individuos y los arrastran hacia prácticas patológicas. La argumentación estrictamente causal concluye, consecuentemente, que «es la constitución moral de la sociedad la que fija en cada momento el contingente de muertos voluntarios», pues existe en cada caso "una fuerza colectiva, de una energía determinada, que empuja a los hombres a que se maten» (ibid.: 336).

Como se puede comprobar, la explicación es situacional y causal. Se puede resumir así: a) en situaciones estructurales de inadecuada regulación y/o integración, b) emergen corrientes sociales de opinión de intensidad variable, pero externas y dotadas de fuerza propia, que $c$ ) penetran en los individuos y los arrastran hacia prácticas suicidas. De este modo, la agregación estadística de conductas individuales se convierte - porque lo es causalmente-en un hecho social sui generis, como había asegurado Durkheim desde el principio.

No quiero entrar a evaluar en su conjunto esta propuesta de conversión que hoy en día nos puede parecer tan burdamente positivista, innecesaria e insostenible. Lo que quiero resaltar es que, incluso en los términos de Durkheim, parece insuficiente. En efecto, la argumentación explicativa ha conseguido asignar una causa a las variaciones de la tasa de suicidios, pero todavía no ha sido capaz de aclarar su condición de inteligibilidad, es decir, especificar cómo opera tal causa sobre su efecto. Su efecto es un determinado actuar humano y, por lo tanto, para que actúe como causa habrá que especificar cómo la corriente opera sobre el agente.

Para resolver este problema de la agencialidad y la condición de inteligibilidad, Durkheim recurre a lo que constituye una constante, ciertamente proteica, en su obra: la idea de la doble naturaleza humana ${ }^{28}$. El agente que recibe el

28 Aparece en su primera formulación en DTS como teoría de las «dos conciencias» (Durkheim, 1893: 74) y se mantiene, con significativos cambios de énfasis, hasta sus últimos trabajos. Para llamar la atención sobre la enorme importancia de lo que acabará llamando el homo duplex, Durkheim publicará un importante artículo sobre el tema. Allí se asegura que «el yo no puede 
impacto de la corriente de malestar colectivo es un ser doble: un sí mismo que es distinto de sí, pues contiene a "la misma sociedad encarnada» (ibid.: 227); «un hombre físico al que se agrega el hombre social» (ibid.: 228). Esta duplicidad, subraya Durkheim, no es pacífica sino tensa, dramática, pues a la «doble existencia» corresponde una "doble tendencia" que supone "dos fuerzas antagónicas [...] en presencia» (ibid.: 360). Y esta duplicidad marca al ser humano en sus dos facultades fundamentales: las ideacional-reflexivas y las prácticopasionales — que denomina «funciones representativas» $\mathrm{y}$ "funciones activas $\mathrm{y}$ prácticas» (ibid.: 440)—. En términos ideacional-reflexivos, el hombre es una autoconciencia individual que participa en una heteroconciencia grupal. En términos práctico-pasionales, actúa movido por sus impulsos pero participa en las tipificaciones sociales de necesidades y medios. Tal duplicidad es constitutiva y se erige en la condición de posibilidad de toda vida humana. Sólo en su marco se puede resolver adecuadamente el problema vital de la determinación de las «necesidades legítimas» y el «sentido de nuestros esfuerzos» (ibid.: 444), es decir, el problema humano de la buena vida.

He aquí en términos muy sintéticos las tesis básicas sobre la dualidad de la naturaleza humana. En este contexto no interesan en sí, sino sólo en relación al problema de la explicación de las variaciones de la tasa social de suicidios. La conexión que proporciona Durkheim es muy directa: dada la duplicidad tensional de los agentes, éstos no pueden resolver sus problemas vitales si no hay una correcta encarnación de lo social en ellos. Tal encarnación puede ser excesiva, en cuyo caso se da lugar a prácticas altruista-fatalistas; o puede ser defectuosa, en cuyo caso se desatan prácticas anómico-egoístas. En razón de ello, Durkheim puede cerrar su argumentación explicativa: las causas sociales son interiores a los agentes; no se limitan a arrastrarlos desde fuera, sino que los conforman desde dentro. El suicida que decide darse muerte lo decide realmente pues es agente de sus actos en la medida en que, en sí mismo, es siempre y a la vez él mismo y otro. Ese otro en el interior de sí es la sociedad. De esta manera se resuelve el problema de la agencia y se cumple con la condición de inteligibilidad.

Así concluye el trayecto teórico: el círculo se cierra. El itinerario inicial ha permitido construir un marco analítico que se puede acomodar y sobreponer a un esquema explicativo. Este esquema cumple con el objetivo explícito de SU: «la tasa social de suicidios no se explica más que sociológicamente» (ibid.: 336), es decir, a partir de otros hechos sociales. Quod erat demostrandum.

ser total y exclusivamente él mismo [soi-même]» y que «hay en nosotros algo distinto de nosotros mismos [autre chose que nous]» que hace que «jamás estemos completamente de acuerdo con nosotros mismos [...] y nos obliga a sobrepasarnos [...], lo que no es posible sin una tensión penosa» (Durkheim, 1914: 319, 320, 331). Esta escisión tensional del yo estaba ya planteada en las páginas de SU y dinamiza lo que más adelante denomino modelo del equilibrio dramático. 


\section{DURKHEIM EN SU LABERINTO}

«Se comulga en la tristeza cuando ya no se tiene otra cosa que poner en común» (ibid.: 230). Este bello florilegio durkeimiano no consigue espantar las dificultades de fondo en las que ha embarrancado la argumentación teórica de SU. Introducido en la argumentación sobre el suicidio egoísta, muestra en realidad que, a pesar de la habilidad literaria de Durkheim y de su dominio de la retórica, se ha creado un laberinto lógico del que no se puede salir. Pues, en efecto, ¿cómo explicar causalmente, por medio de las corrientes sociales de desánimo y tristeza que penetran desde fuera, la conducta suicida del agente si para hacerla inteligible se ha propuesto la ausencia de lo social en su interior? Tal es la lógica del suicidio egoísta: causalmente y como hecho sui generis responde a condiciones estructurales de defectuosa integración social y es producto de corrientes de opinión que arrastran a los agentes; pero desde el punto de la inteligibilidad (cómo actúan tales causas sobre los agentes) presupone el vacío de lo social en el interior del suicida y, sólo en razón de tal ausencia, se hace inteligible su conducta. En consecuencia, lo que muestra su poder causal no es la presencia presionante de algo, sino su ausencia.

Para aclarar estas dificultades y los laberintos que de ellas surgen es preciso volver a recorrer en un nivel de abstracción mayor el itinerario teórico que se presupone en SU. Si atendemos al itinerario analítico encontramos que lo que Durkheim propone como condiciones de posibilidad del orden social se enuncia en términos de un modelo de equilibrio. Este modelo privilegia como ordenado un punto medio que se halla en algún lugar entre dos extremos de desorden, pues «en el orden vital nada es bueno sin mesura» (ibid.: 233). En términos de la tradicional teoría moral, tal modelo privilegia lo que los antiguos llamaban la prudencia como habilidad práctica para situarse en un punto intermedio entre los extremos que inestabilizan el mundo de la acción ${ }^{29}$. Podemos denominarlo, por tanto, modelo de equilibrio de la prudencia. Volcado ya en el campo de la explicación, ese modelo sigue operando, pero encuentra interesantes cualificaciones. En efecto, a la hora de dar cuenta de las condiciones de posibilidad de orden social en las sociedades históricas concretas, Durkheim vuelve sobre el modelo del equilibrio, pero no ya como prudencia del punto medio de la virtud, sino en el sentido de la física, como equilibrio entre fuerzas dispares que se contrarrestan y definen un punto estable de compensación ${ }^{30}$. Pasamos, pues, del equilibrio como prudencia al equilibrio físico como compensación de fuerzas dispares. En ambos casos, el equilibrio se pre-

29 Sobre prudencia, acción y buena vida en la filosofía práctica y en la tragedia griega, véase el extraordinario trabajo de Nussbaum (1995).

${ }^{30}$ Un texto representativo de esta variante del equilibrio es el siguiente: «no hay ideal moral que no combine, en proporciones variables según sociedades, el egoísmo, el altruismo y una cierta anomia [...] Allí donde se atemperan mutuamente, el agente moral se halla en un estado de equilibrio» (Durkheim, 1897: 363). Las ideas que subyacen son dos: lo social como co-presencia de fuerzas con dirección distinta; el orden como equilibrio en el que esas fuerzas se compensan. 
senta como un hecho social en el que sólo intervienen mecanismos y fuerzas de ese tipo.

Esta apuesta por el modelo del equilibrio da lugar a una tercera variante. Podemos denominarla variante dramática del equilibrio, pues lo que la caracteriza es que el estado de orden es un resultado precario producto de la contraposición de dos fuerzas radicalmente heterogéneas: la hybris individual y el destino social — no se trata, por lo tanto, de un juego entre fuerzas sociales-. Es evidente que no se trata de la terminología de Durkheim, pero a estos términos clásicos es traducible la contraposición de un individuo que se ensimisma, «enfebrece y exaspera» (ibid.: 440) y/o desea al deseo, cayendo en "la sed de lo que no es» (ibid.: 304), enfrentándose a una fuerza social que le proporciona un sentido colectivo a su acción y objetivos legítimos alcanzables. En el primer caso estamos ante la hybris individual que lleva a la destrucción; en el segundo, ante la asunción de un destino que corresponde a algún designio cósmico - pues, como vimos, la sociedad forma parte del complejo de fuerzas cósmicas.

Este triple modelo del equilibrio (prudencial, físico y dramático) domina el discurso teórico de Durkheim, tanto en lo que tiene de construcción analítica como de aplicación-especificación explicativa. En paralelo a él corre otro, también de orden muy abstracto, que, a falta de mejor etiqueta, denominaré de la intrincada dialéctica del espacio. Se edifica sobre una doble distinción crucial: individuo/sociedad y presencia/ausencia. La primera distinción es pensada en términos del modelo dramático de equilibrio: sociedad e individuo son espacios segregados y enfrentados; así como la sociedad se resiste a la individuación, el individuo se resiste a la socialización. De la lógica del espacio se pasa a la lógica del equilibrio dramático. Las cosas se complican en razón de la interferencia de la segunda distinción espacial: aquella que separa lo ausente y lo presente. Pues la argumentación de Durkheim sobre la doble naturaleza del hombre lleva a concebir el espacio segregado del individuo a la vez como presencia y ausencia de lo social. En razón de ello, el espacio individual está a la vez segregado de, integrado en, o penetrado por, lo social. Tal ausencia/presencia es pensada en términos de las distintas variantes del modelo del equilibrio: presencia/ausencia prudente; presencia/ausencia compensada o física; presencia/ausencia dramática. Esta última es la más relevante y es la que, a la hora de dar cuenta de las prácticas suicidas características de las sociedades contemporáneas, genera insalvables dificultades lógicas. Pues el diagnóstico de Durkheim sobre anomia y egoísmo propone a la vez la ruptura del equilibrio dramático en perjuicio de uno de los polos (la sociedad) y la consecuente ausencia de lo social en el espacio segregado de lo individual. Si es así, entonces lo ausente (social) no puede explicar las prácticas individuales, ya que explícitamente se niega su presencia en el interior del individuo. El suicida es siempre retratado como paradigma de ser desocializado. En razón de ello, la corriente social, que supone presencia para poder ser causa, ha de ser desechada como tal causa para poder hacer inteligible la práctica anómico-egoísta del agente.

Alcanzamos así un laberinto lógico en cuyos pasadizos se pierde el hilo que 
habría de conectar las causas sociales con las prácticas inteligibles de los agentes. Según el principio de causalidad, lo ausente (social) ha de estar presente, pues sólo así se pueden imputar a las corrientes sociales las prácticas de los agentes. Pero, según el principio de inteligibilidad, lo presente (social) ha de estar ausente, pues sólo así se puede explicar el desvarío de sentido y deseo que lleva al suicidio. Ausente y presente a la vez, lo social no puede cumplir el cometido que se le asigna: la explicación de las variaciones de la tasa social de suicidios como hecho sui generis. Esto lleva a que el modelo del equilibrio sea sociológicamente operativo en sus variantes prudencial y física, ya que entonces sí consigue explicar socialmente lo social. Pero en su variante última, la del equilibrio dramático, deja de ser propiamente sociológico al modo en que Durkheim piensa lo sociológico. La razón es obvia: pudiendo dar cuenta de la ausencia/presencia, no puede explicar socialmente las prácticas de los agentes.

\section{SOBRE LA FRAGILIDAD DEL TÓTEM}

Durkheim dejó dicho que un tótem proporciona continuidad fundacional a las prácticas sociales: es legítimo que hagamos lo que hacemos porque ya lo hicieron, de forma ejemplar, nuestros ancestros. Desde este punto de vista, todo tótem se asienta en la piedad colectiva. Bastaría la impiedad para deshacerlo.

Las prácticas académicas de interpretación textual son siempre ambivalentes: pías e impías a la vez. La piedad viene del reconocimiento previo de la relevancia del texto. La impiedad, de su conversión en un objeto diseccionable. El resultado final nada entre estas dos aguas: el homenaje es también ultraje.

Hemos visto que, en uno de sus aspectos cruciales, SU es un tótem frágil. Traza un círculo virtuoso para acabar desembocando en un laberinto. Dado el prestigio académico de la lógica, un diagnóstico tal parece desembocar en la desacralización del tótem; y un tótem sin sacralidad se convierte en rareza museística. ¿Debemos enviar SU al museo de las rarezas sociológicas? No es el caso. SU sigue mostrándose una obra original, rica, compleja, buena food for thought, muy por encima de otros legados de la tradición. Lo que debemos hacer es reconocer sus fragilidades, convertir, al modo propuesto por Latour, el fetiche en un fatiche $e^{31}$ que, a medio camino entre el frágil constructo artificial inmanente y prejuiciado y el reverenciado monumento trascendente y verdadero, se sitúa en el orden de las objetivaciones más auténticas del difícil proceso de la práctica del conocimiento. Tótem frágil, SU sigue siendo un punto de referencia crucial de nuestra tradición.

31 Traduzco así el juego de palabras de Latour (1996), que contrapone fétiche (fetiche) y faitiche (fatiche), explotando la probable derivación del feitiço portugués - de donde viene fetiche- de feito (un hecho y lo que está hecho o fabricado). A diferencia de un fetiche, un fatiche hace referencia, propone Latour, a «la robusta certidumbre que permite que la práctica pase a la acción sin creer jamás en la diferencia entre lo que se construye y lo que se recoge, lo inmanente y lo trascendente» (Latour, 1996: 44). 


\section{BIBLIOGRAFÍA}

Alexander, J. C. (1982): The Antinomies of Classical Thought: Marx and Durkheim, London, Routledge.

CoHen, B. (1990): «Scientific revolutions, revolutions in science and a probabilistc revolution 1800-1930», en L. Krüger, L. Daston y M. Heidelberg (eds.), The Probabilistic Revolution. Vol. 1: Ideas in History, Cambridge, MIT Press, pp. 23-44.

Coser, L. A. (1971): Masters of Sociological Thought. Ideas in Historical and Social Context, New York, Harcourt Brace Jovanovich.

Baudelot, C., y Establet, R. (1984): Durkheim et le suicide, París, PUF.

Besnard, P. (1973): «Durkheim et les femmes ou Le Suicide inachevé», Revue Française de Sociologie, 14: 27-61.

- (1978): «Merton à la recherche de l'anomie», Revue Française de Sociologie, 19: 3-38.

- (1987): L'anomie, ses usages et ses fonctions dans la discipline sociologique depuis Durkheim, París, PUF.

- (1993): "Anomie and fatalism in Durkheim's theory of regulation», en S. Turner (ed.), Emile Durkheim. Sociologist and Moralist, London, Routledge, pp. 169-190 (traducido en este volumen).

Borlandi, M. (1994): "Informations sur la rédaction du Suicide et sur l'état du conflit entre Durkheim et Tarde de 1895 à 1897», Durkheim Studies, 6: 4-13 (traducido en este volumen).

DASTON, L. (1990): «Rational individuals versus laws of society: from probability to statics», en L. Krüger, L. Daston y M. Heidelberg (eds.), The Probabilistic Revolution. Vol. 1: Ideas in History, Cambridge, MIT Press, pp. 295-304.

Dohrenwend, B. P. (1959): «Egoism, altruism, anomie and fatalism: a conceptual analysis of Durkheim's types", American Sociological Review, 24: 466-472.

Douglas, J. D. (1966): «The sociological analysis of social measuring of suicide», Archives Européennes de Sociologie, 7: 249-275.

Durkheim, E. (1893): De la division du travail sociale, París, Alcan (citas por la edición PUF, París, 1973).

- (1895): Les règles de la méthode sociologique, París, Alcan (citas por la edición PUF, París, 1973).

- (1897): Le suicide, París, Alcan (citas por la edición PUF, París, 1973).

- (1902): «Sur le totémisme», L'Année Sociologique, 5: 82-121 (citas por E. Durkheim, Le Journal Sociologique, París, PUF, 1969).

- (1914): "Le dualisme de la nature humaine et ses conditions sociales», Scientia, 15: 206-221 (citas por E. DurKheIm, La science sociale et l'action, París, PUF, 1970, pp. 314-332).

ELSTER, J. (1991): Tuercas y tornillos. Una introducción a los conceptos básicos de las ciencias sociales, Barcelona, Gedisa.

Ewald, F. (1996): Histoire de l'État Providence, París, Grasset.

GidDENS, A. (1965): "The suicide problem in French sociology», British Journal of Sociology, 16: $1-18$.

- (1996): "A typology of suicide», European Journal of Sociology, 7: 276-295 [en Hamilton (ed.), Emile Durkheim. Critical Assesments, London, Routledge, 1990, III, pp. 99-119].

Halbwachs, M. (1930): Les causes du suicide, París, Alcan.

Hamilton, P. (ed.) (1990): Emile Durkheim. Critical Assesments, vols. I-IV, London, Routledge.

- (1995): Emile Durkheim. Critical Assesments. Second Series, vols. V-VIII, London, Routledge.

Horton, J. (1964): «The deshumanization of anomie and alienation: a problem in the ideology of sociology", British Journal of Sociology, 15: 283-300.

Johnson, B. D. (1965): "Durkheim's one cause of suicide», American Journal of Sociology, 30: 875-886.

La Capra, D. (1972): Emile Durkheim, New York, Cornell University Press.

Latour, B. (1996): Petite réflexion sur le culte moderne des dieux faitiches, París, Synthelabo Groupe. 
LÉCUYER, B.-P. (1990): «Probability in vital and social statistics: Quetelet, Farr and the Bertillons», en L. Krüger, L. Daston y M. Heidelberg (eds.), The Probabilistic Revolution. Vol. 1: Ideas in History, Cambridge, MIT Press, pp. 317-335.

Lukes, S. (1984): Emile Durkheim. Su vida y su obra, Madrid, CIS.

MAWson, A. R. (1970): "Durkheim and contemporary social pathology», British Journal of Sociology, 21: 298-313.

Merton, R. K. (1970): Teoría y estructura sociales, México, FCE.

Mestrovic, S. G. (1987): "Durkheim's concept of anomie considered as a total social fact», British Journal of Sociology, 38: 567-583.

- (1988a): «Durkheim, Schopenhauer and the relationship between goals and means: reversing the assumptions in the parsonian theory of rational action", Sociological Inquiry, 58: 163-181 [recogido en P. Hamilton (ed.), Emile Durkheim. Critical Assesments. Second Series, London, Routledge, 1995, VIII, pp. 287-304].

- (1988b): "The social world as Will and Idea: Schopenhauer's influence upon Durkheim's thought», Sociological Review, 36: 674-705 [recogido en P. Hamilton (ed.), Emile Durkheim. Critical Assesments. Second Series, London, Routledge, 1995, VIII, pp. 305-329].

Mestrovic, S. G., y Brown, H. M. (1985): «Durkheim's concept of anomie as dérèglement», Social Problems, 33: 81-99.

Nussbaum, M. (1995): La fragilidad del bien. Fortuna y ética en la tragedia y la filosofía griega, Madrid, Visor.

Oberschall, A. (1990): "The two empirical roots of social theory and the probability revolution», en L. Krüger, G. Gigerenzer y M. S. Morgan (eds.), The Probabilistic Revolution. Vol. 2: Ideas in the Science, Cambridge, MIT Press, pp. 103-31.

Orru, M. (1983): "The ethics of anomie: Jean Marie Guyau and Emile Durkheim», British Journal of Sociology, 34: 499-518.

Overington, M. A. (1981): «A rhetorical appreciation of a sociological classic: Durkheim's Suicide», Canadian Journal of Sociology, 6: 447-462 (traducido en este volumen).

PARSONS, T. (1968): La estructura de la acción social, Madrid, Guadarrama.

PAul-LÉvy, F. (1987): «La mesure du bio-social», Cahiers Internationaux de Sociologie, 83: 281 289 [recogido en P. Hamilton (ed.), Emile Durkheim. Critical Assesments. Second Series, London, Routledge, 1995, VIII, pp. 243-248].

Pope, W. (1976): Durkheim's Suicide. A Classical Analysed, Chicago, University of Chicago Press.

Porter, T. M. (1990): «Lawless society: social science and the reinterpretation of statistics in Germany, 1850-1880», en L. Krüger, L. Daston y M. Heidelberg (eds.), The Probabilistic Revolution. Vol. 1: Ideas in History, Cambridge, MIT Press, pp. 350-375.

Ramos, R. (1982): «Introducción» a E. Durkheim, El socialismo, Madrid, Editora Nacional, pp. 9-96.

- (1996): "Los saberes del patriarca: Emile Durkheim y el suicidio de las mujeres», en M. A. Durán (ed.), Mujeres y hombres en la formación de la teoría sociológica, Madrid, CIS, pp. 6572.

SELKIN, J. (1983): "The legacy of Emile Durkheim», Suicide and Life Threatening Behavior, 13: 3-14 [en Hamilton (ed.), Emile Durkheim. Critical Assesments, London, Routledge, 1990, III, pp. 200-210].

SROle, L. (1956): "Social integration and certain corollaries: an explanatory study», American Sociological Review, 21: 709-716.

Tilly, C. (1991): Grandes estructuras, procesos amplios, comparaciones enormes, Madrid, Alianza.

Tiryakian, E. (1981): «Sexual anomie, social structure, societal change», Social Forces, 59: 10251053.

VAN PARIJS, P. (1990): Le modèle économique et ses rivaux. Introduction à la pratique de l'épistémologie des sciences sociales, París, Droz. 


\begin{abstract}
Suicide can be considered one of the totems of contemporary sociology. Most of its power comes from its theoretical density. However, its theoretical framework is complex and is an object of interpretative debates. This article proposes a reconstruction of Suicide's theoretical logic as the outcome of a round path that first breaks down its object analitically and then turns to it to make it intelligible. It is then when serious apories come out. These confront the causal explanation principle with the intelligibility principle of analysed practices. From that it follows the theoretical fragiliy of Suicide.
\end{abstract}

